\title{
Heavy Flavour Physics with the ALICE Muon Spectrometer at the LHC
}

\author{
Xiaoming Zhang for the ALICE Collaboration* \\ Laboratoire de Physique Corpusculaire, Clermont Université, Université Blaise Pascal, \\ CNRS-IN2P3, Clermont-Ferrand, France \\ Central China Normal University, Institute of Particle Physics, Wuhan, China \\ E-mail: xiaoming.zhang@clermont.in2p3.fr
}

\begin{abstract}
ALICE is the dedicated heavy ion experiment at the LHC. The main purpose of the experiment is to investigate the properties of strongly interacting matter at extreme energy density where the formation of the Quark Gluon Plasma is expected. In this regard, heavy flavours which are produced in the early stage of the collision and coexist with the surrounding medium are especially relevant. The successful achievement of the heavy-ion program requires also the study of proton-proton collisions. Besides providing the necessary baseline for nucleus-nucleus collisions, proton-proton collisions allow to test perturbative QCD in a new energy regime. In ALICE, heavy flavours are measured through the electron channel and the hadron channel in the central barrel as well as through the muon channel in the forward muon spectrometer. During the 2010 data taking period, ALICE has recorded proton-proton collisions at $\sqrt{s}=0.9 \mathrm{TeV}, 2.36 \mathrm{TeV}$ and $7 \mathrm{TeV}$ and, $\mathrm{Pb}-\mathrm{Pb}$ collisions at $\sqrt{s_{N N}}=2.76 \mathrm{TeV}$. In this contribution, special attention is given to the first physics results on heavy flavour production in $p-p$ collisions at $\sqrt{s}=7 \mathrm{TeV}$ measured with the ALICE muon spectrometer in the forward region.
\end{abstract}

XLIX International Winter Meeting on Nuclear Physics

Bormio, Italy

January, 24-28, 2011

${ }^{*}$ Speaker. 


\section{Introduction}

The LHC (Large Hadron Collider) collided protons at a center of mass energy $\sqrt{s}=7 \mathrm{TeV}$ and lead ions at a center of mass energy per nucleon pair $\sqrt{s_{N N}}=2.76 \mathrm{TeV}$. This energy which exceeds the one reached at RHIC for Au-Au collisions by about a factor 14 will provide new insights for the study of the properties of strongly interacting matter under extreme thermodynamical conditions [1]. One of the most important aspect of this new energy range is the abundant production rate of heavy quarks (charm and beauty). Expected yields and cross sections of $c \bar{c}$ and $b \bar{b}$ pairs calculated in the framework of perturbative QCD (pQCD) at next-to-leading-order (NLO) are reported in Table 1 for the 5\% most central $\mathrm{Pb}-\mathrm{Pb}$ collisions at $\sqrt{s_{N N}}=2.76 \mathrm{TeV}$ and for $p$ - $p$ collisions at $\sqrt{s}=7 \mathrm{TeV}$ (calculated from [2]).

\begin{tabular}{|c|c|c|c|c|}
\hline System & $N_{c \bar{c} / \text { event }}$ & $\sigma_{c \bar{c}}^{N N}(\mathrm{mb})$ & $N_{b \bar{b} / \text { event }}$ & $\sigma_{b \bar{b}}^{N N}(\mathrm{mb})$ \\
\hline $\mathrm{Pb}-\mathrm{Pb}(2.76 \mathrm{TeV})$ & 56 & 2.1 & 2 & 0.075 \\
$\mathrm{p}-\mathrm{p}(7 \mathrm{TeV})$ & 0.10 & 6.9 & 0.003 & 0.23 \\
\hline
\end{tabular}

Table 1: ALICE baseline for heavy quark production cross sections and yields in $p$ - $p$ collisions $(\sqrt{s}=7$ $\mathrm{TeV}$ ) and in central $\mathrm{Pb}-\mathrm{Pb}$ collisions $\left(\sqrt{s_{N N}}=2.76 \mathrm{TeV}\right)$. For $\mathrm{Pb}-\mathrm{Pb}$ collisions, nuclear shadowing is included and binary scaling is applied.

Heavy quarks are produced at early stages of the collision and experience the full evolution of the medium, thus allowing to investigate their production mechanisms, propagation and hadronization in the hot and dense nuclear medium. In addition, the large charm and beauty cross sections allow to investigate new observables $[3,4]$ for the study of heavy quark quenching. Theoretical calculations have shown that the ratios of the nuclear modification factor of $D(B)$-hadrons to that of light hadrons are promising observables to probe the color charge (mass) dependence of parton energy loss and the ratio of beauty to charm nuclear modification factor is expected to allow to isolate the mass dependence of the energy loss. The successful achievement of the heavy ion program requires also the study of $p$ - $p, p$-A and light A-A systems. Besides providing the necessary baseline for the study of medium effects in nucleus-nucleus collisions, $p$ - $p$ collisions are of crucial interest as an important test of QCD in a new kinematic region of unprecedented small Bjorken- $x$ values down to about $10^{-5}$ [5]. Note that pQCD calculations can be used to scale the charm and beauty cross sections in $p-p$ collisions, measured at $\sqrt{s}=7 \mathrm{TeV}$, to the $\mathrm{Pb}-\mathrm{Pb}$ nucleon-nucleon center of mass energy of $2.76 \mathrm{TeV}$. The measurement of charm and beauty cross sections is also important for understanding quarkonium production. Finally, it is worth pointing out that the measurement of the beauty production cross section is mandatory to estimate the contribution of secondary $J / \psi$ from $B$-hadron decay to the total $J / \psi$ yield.

Heavy flavours are measured in ALICE with (di)electrons and through the hadronic decay channel at central rapidities and, with (di)muons at forward rapidities. The LHC delivered its first proton beams in November 2009 and collided lead ions at $\sqrt{s_{N N}}=2.76 \mathrm{TeV}$ at end of 2010. Protonproton collisions have been measured at $\sqrt{s}=0.9 \mathrm{TeV}, \sqrt{s}=2.36 \mathrm{TeV}$ and $\sqrt{s}=7 \mathrm{TeV}$. First results obtained in the mid-rapidity region can be found in $[6,7,8,9,10,11,12,13]$. In the following, first results on heavy flavour production in $p$ - $p$ collisions at $\sqrt{s}=7 \mathrm{TeV}$, via single muons measured with the ALICE muon spectrometer are discussed. 


\section{The ALICE muon spectrometer}

A detailed description of the ALICE apparatus can be found in [14]. The ALICE detector consists of a central barrel (pseudo-rapidity range: $|\eta|<0.9$ ) placed in the LEP L3 magnet (B $\leq 0.5 \mathrm{~T})$, a forward muon spectrometer and several small angle acceptance sub-detectors in the forward and backward pseudo-rapidity regions. Amongst those, the VZERO detector is used in the present analysis (see sub-section 3.1). It consists of two scintillator arrays placed at each side of the interaction point and covering $2.8<\eta<5.1$ and $-3.7<\eta<-1.7$. The main detectors in the central barrel are, the Inner Tracking System, the Time Projection Chamber, the Transition Radiation Detector for electron identification, the Electromagnetic Calorimeter for high momentum photon and electron measurement and the Time of Flight system for identification of hadrons at high transverse momentum.

The purpose of the ALICE muon spectrometer is the study of quarkonium production and heavy flavour production in the (di)muon channel. In addition, the production of weakly interacting probes ( $Z^{0}$ and $W^{ \pm}$bosons) and low mass resonances $(\rho, \omega, \phi)$ is also investigated. The main design criteria are driven by the requirements that the detector should operate in the high multiplicity environment of central $\mathrm{Pb}-\mathrm{Pb}$ collisions at $\sqrt{s_{N N}}=5.5 \mathrm{TeV}$ and should reach a mass resolution of $100 \mathrm{MeV} / c^{2}$ in the $\Upsilon$ mass region $\left(\mathrm{M} \sim 10 \mathrm{GeV} / \mathrm{c}^{2}\right)$ in order to resolve the $\Upsilon(1 S), \Upsilon(2 S)$ and $\Upsilon(3 S)$ states.

The muon spectrometer covers $-4.0<\eta<-2.5$ (polar angular range: $171^{\circ}<\theta<178^{\circ}$ ). It is composed of a passive front absorber made of composite materials (carbon, concrete and steel), a beam shield, a $3 \mathrm{~T} \cdot \mathrm{m}$ dipole magnet, five stations of high granularity tracking chambers, each based on two planes of Cathod Pad Chambers. Finally, two stations of trigger chambers equipped with two planes of Resistive Plate Chambers each, are located downstream of the tracking system, behind a $1.2 \mathrm{~m}$ thick iron wall. The front absorber and the muon filter wall stop muons with momentum lower than $4 \mathrm{GeV} / \mathrm{c}$. Most of punch-through hadrons (charged hadrons that penetrate the front absorber without suffering a hadronic interaction) are stopped in the muon filter. The trigger system allows to reject background muons from $\pi$ and $K$ decays by means of a transverse momentum $\left(p_{\mathrm{t}}\right)$ cut on single tracks. Low $p_{\mathrm{t}}(\sim 1 \mathrm{GeV} / \mathrm{c})$ and high $p_{\mathrm{t}}(\sim 1.7 \mathrm{GeV} / \mathrm{c})$ trigger cuts have been optimized for the measurement of charmonia and bottomonia, respectively.

\section{Measurement of the production of muons from heavy flavour decay in $p-p$ collisions at $\sqrt{s}=7 \mathrm{TeV}$ with the ALICE muon spectrometer}

\subsection{Experimental conditions and data sample}

The ALICE experiment has measured $p$ - $p$ collisions at $\sqrt{s}=7 \mathrm{TeV}$ for minimum bias collisions with at least one charged particle in 8 units of pseudo-rapidity (INT1B trigger) and events with at least one muon in the muon spectrometer (MUS1B trigger) with a transverse momentum larger than $\sim 0.5 \mathrm{GeV} / \mathrm{c}$. The INT1B trigger requires at least one signal in the Silicon Pixel Detector (SPD) or in one of the two VZERO counters placed on both sides of the interaction point, in coincidence with the two beam counters. The MUS1B trigger requires hits in at least three (out of four possible) trigger chamber planes. In the bending plane (plane perpendicular to magnetic field), the track deviation relative to a straight line is computed. By applying a cut on this deviation, one 
can select tracks above a given $p_{\mathrm{t}}$ threshold. In the considered data taking period, the $p_{\mathrm{t}}$ threshold is $\sim 0.5 \mathrm{GeV} / \mathrm{c}$

A higher statistics of muon tracks is collected with MUS1B trigger events as compared to the INT1B trigger events. As shown in Fig. 1, $p_{\mathrm{t}}$ and $\eta$ distributions of muon tracks (selected by a set of cuts which will be described in what follows) exhibit a same behaviour for MUS1B and INT1B trigger events, as expected.
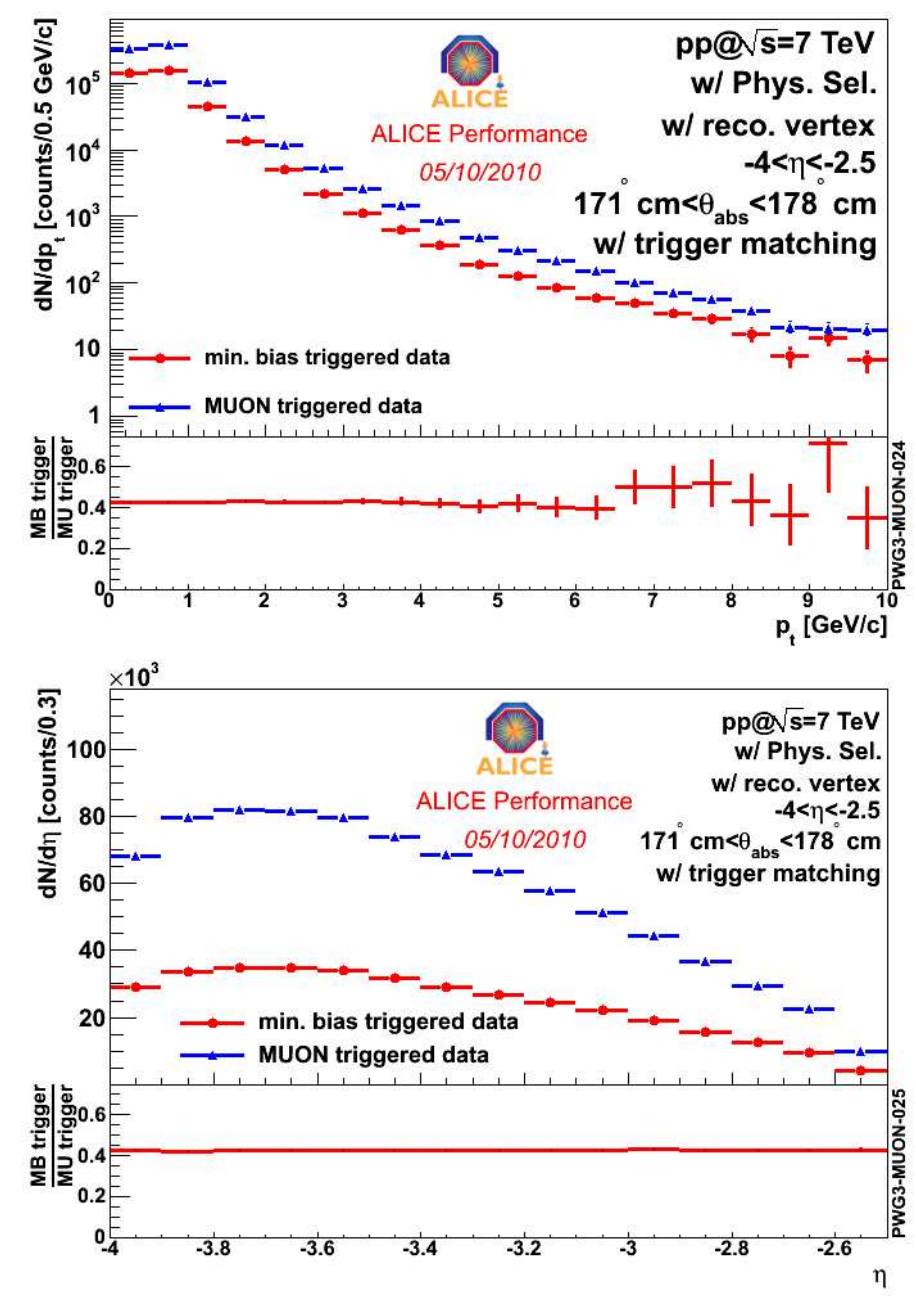

Figure 1: Single muon $p_{\mathrm{t}}$ (upper panel) and $\eta$ (lower panel) distributions for MUS1B (blue) and INT1B (red) trigger in $p$ - $p$ collisions at $\sqrt{s}=7 \mathrm{TeV}$. The ratio of the distributions obtained with the two triggers is displayed at the bottom of each figure.

The single muon analysis is carried out for MUS1B trigger events while, as it will be discussed in sub-section 3.4, minimum bias data are used for normalization, only.

The luminosity in ALICE was kept to values of $[0.6-1.2] \cdot 10^{29} \mathrm{~cm}^{-2} \mathrm{~s}^{-1}$ in order to keep the collision pile-up rate in the same bunch crossing below 5\%. The alignment of the tracking chambers, a crucial step for the single muon analysis, has been carried out using the MILLEPEDE package [15], by analyzing tracks without magnetic field in the dipole. The corresponding resolu- 
tion in the bending plane ranges between $300 \mu \mathrm{m}$ and $900 \mu \mathrm{m}$, depending on $\eta-\varphi$ position. This leads to a relative systematic uncertainty on $p_{\mathrm{t}}$ measurement of about $2 \%$ which limits the $p_{\mathrm{t}}$ reach to $10 \mathrm{GeV} / \mathrm{c}$. This alignment has been improved recently and is now applied to the whole statistics collected in 2010.

In what follows a sample of about $1.94 \cdot 10^{6}$ MUS1B events is used. The corresponding integrated luminosity is $3.49 \mathrm{nb}^{-1}$. An offline selection has been applied to reduce the beam induced background from $1 \%$ to $0.1 \%$. Moreover, events without a reconstructed primary vertex by the SPD are rejected from the analysis. Various kinematic cuts have been applied for the track selection: tracks are required to be reconstructed within the acceptance of the muon spectrometer $(-4<\eta<-2.5)$ and to have a polar angle at the end of the front absorber $\left(\theta_{\text {abs }}\right)$ ranging from $171^{\circ}$ to $178^{\circ}$. Finally, one requires that the track candidate in the tracking system matches the track reconstructed in the trigger system (this cut will be discussed in next subsection).

\subsection{Background subtraction}

The measurement of the muon yield from heavy flavour decay is based on the inclusive $p_{\mathrm{t}}$ distribution. The different contributions to this distribution have been identified by means of a Monte-Carlo simulation using Pythia event generator. They are displayed in Fig. 2. One can distinguish: muons from charm and beauty decay (red and green histograms), muons from primary light hadron decay (mainly, $\pi$ and $K$ decay, blue histogram), muons from the decay of secondary light hadrons produced inside the front absorber (magenta histogram) and punch-through hadrons (cyan histogram). Unidentified tracks (brown histogram) correspond to reconstructed tracks which cannot be associated to the generated ones.

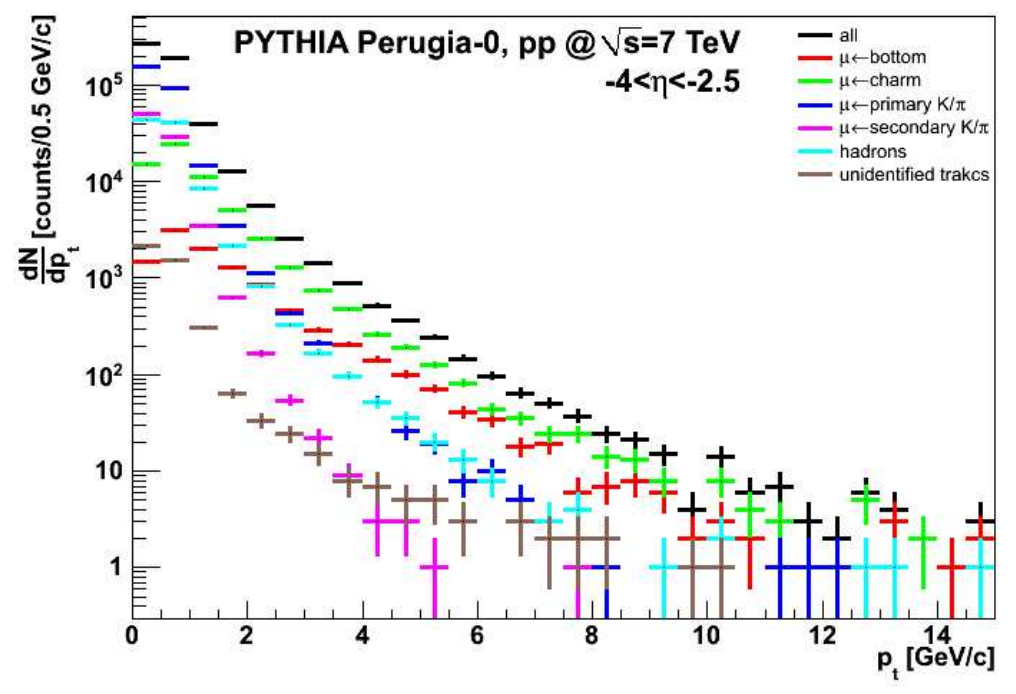

Figure 2: Transverse momentum distribution of reconstructed tracks in the ALICE muon spectrometer (black histogram) in a minimum bias Pythia simulation of $p$ - $p$ collisions at $\sqrt{s}=7 \mathrm{TeV}$.

The trigger system is very effective for rejecting the hadronic component which is stopped in the iron wall. Indeed, as illustrated in Fig. 3, the structure evidenced at high DCA (Distance of Closest Approach: distance between the extrapolated track and the interaction vertex, in the 
plane perpendicular to the beam direction and containing the vertex) is attributed to punch-through hadrons (upper panel) and is suppressed when applying the matching condition between the track measured in the muon chambers and the corresponding track in the trigger system (lower panel).
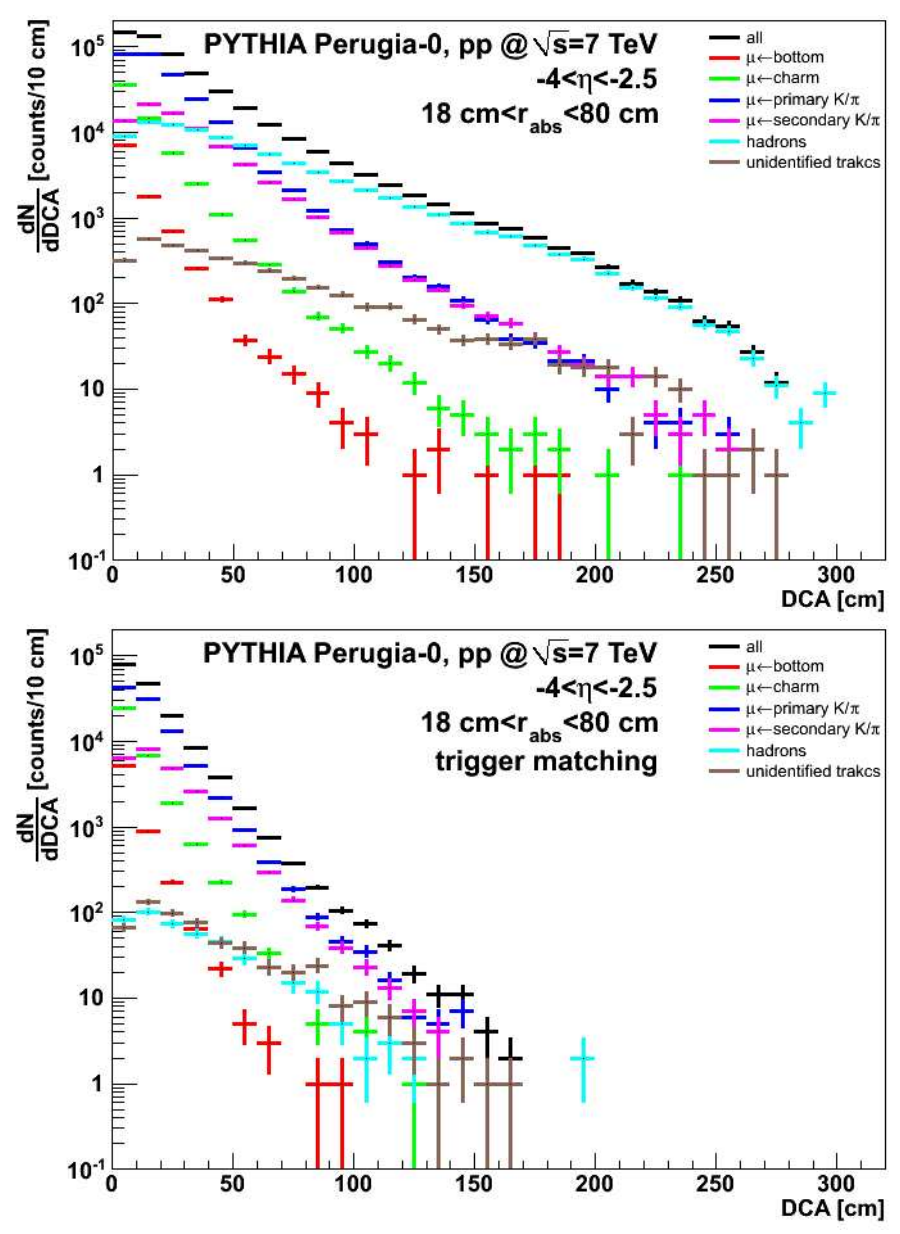

Figure 3: Upper panel: DCA distribution of reconstructed tracks in the ALICE muon spectrometer without requiring the tracking-trigger matching. Lower panel: DCA distribution of reconstructed tracks in the ALICE muon spectrometer when requiring the tracking-trigger matching. The different sources are shown on the figures. These results have been obtained from Pythia simulations of minimum bias $p-p$ collisions at $\sqrt{s}=7 \mathrm{TeV}$.

The experimental DCA distribution (Fig. 4) exhibits the expected trend without (red histogram) and with (blue histogram) the matching between the tracking track and the trigger track (see simulation results in Fig. 3).

In what follows, we focus on the $\mathrm{p}_{\mathrm{t}}>2 \mathrm{GeV} / \mathrm{c}$ range where the contribution of muons from secondary light hadrons is small (about 3\%). Therefore, the main background source consists of muons from the decay of primary pions and kaons and amounts to $25 \%$ of the total yield. Its subtraction is based on full Pythia simulations with realistic conditions. The $p_{\mathrm{t}}$ distribution of muons from primary pions and kaons decay from Pythia (tune Perugia0 [16]), as a function of $\eta$, is assumed to be the same as in the data. Then, the normalization (total muon yield over muons from 


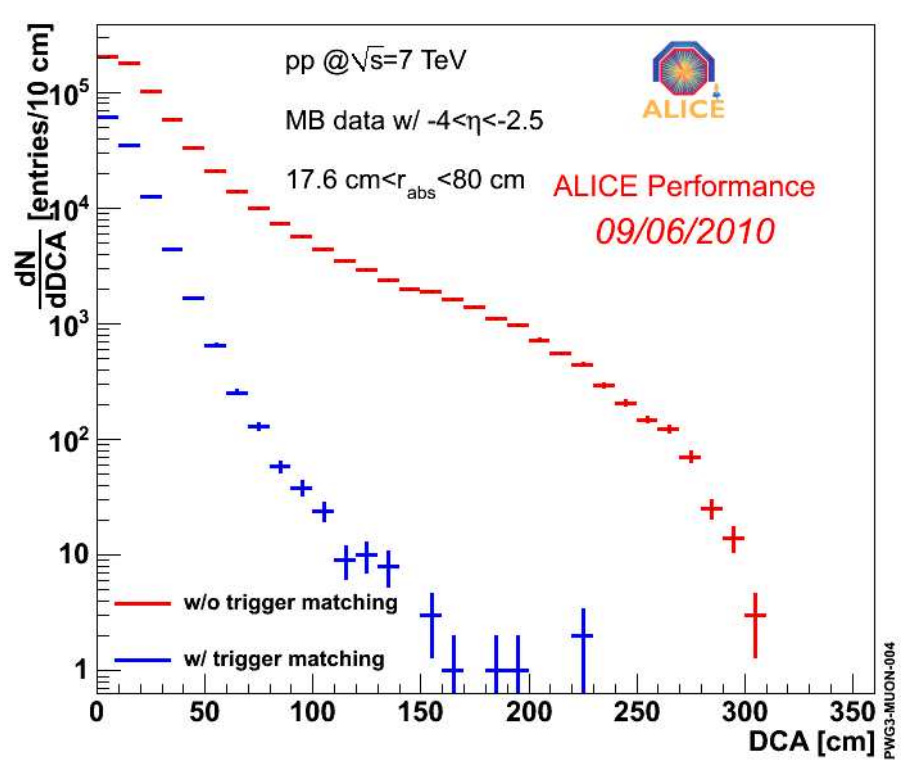

Figure 4: Experimental DCA distribution of single tracks reconstructed in the ALICE muon spectrometer, in $p$ - $p$ collisions at $\sqrt{s}=7 \mathrm{TeV}$. Red (blue) histograms have been obtained without (with) requiring the tracking-trigger matching.

the decay of primary pions and kaons) is done in the low $p_{\mathrm{t}}$ region $\left(0.5<p_{\mathrm{t}}<1.0 \mathrm{GeV} / \mathrm{c}\right)$ where this component is dominant.

The systematic uncertainty on background subtraction from the model input and the transport code (here, Geant3) has been estimated by using different Pythia tuning (ATLAS-CSC [17]) and by varying the yield of muons from the decay of secondary light hadrons within $100 \%$ in the MonteCarlo. The resulting systematic uncertainty ranges from $20 \%$ to $7 \%$ for $p_{\mathrm{t}}$ going from $2 \mathrm{GeV} / \mathrm{c}$ to $6.5 \mathrm{GeV} / \mathrm{c}$. An additional systematic uncertainty of $20 \%$ has been taken into account in order to cover any other effects.

\subsection{Acceptance and efficiency correction}

The $p_{\mathrm{t}}$ distribution of muons from heavy flavour decay is corrected for acceptance and efficiency by means of simulations modeling the full response of the muon spectrometer. The procedure is based on the generation of a large sample of charm (or beauty) signals by using a fast generator of heavy quark pairs based on a parameterization of Pythia calculations. The resulting $p_{\mathrm{t}}$ and $\eta$ acceptance and efficiency correction is displayed in Fig. 5. It is worth noticing that for $p_{\mathrm{t}}>2 \mathrm{GeV} / \mathrm{c}$ the global efficiency is larger than $87 \%$. The systematic uncertainty corresponding to the sensitivity of the acceptance $\times$ efficiency correction on the input $p_{\mathrm{t}}$ and $\eta$ distributions is smaller than $1 \%$ and can be neglected. The main source of systematic uncertainty comes from the accuracy in modeling the detector and is expected to be smaller than $5 \%$.

\subsection{Normalization}

In order to get an inclusive production differential cross section of muons from heavy flavour decay, one needs to normalize the acceptance and efficiency corrected muon yields to the number 


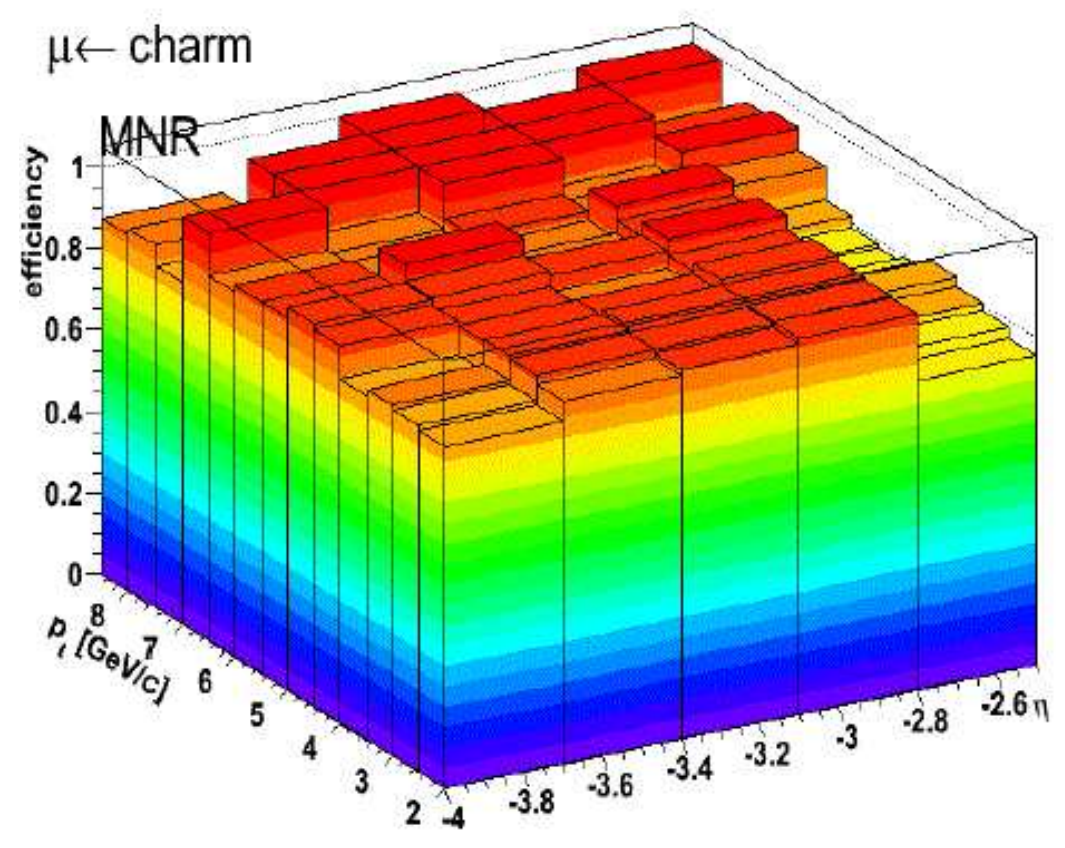

Figure 5: Single muon acceptance $\times$ efficiency as a function of $p_{\mathrm{t}}$ and $\eta$ obtained from a realistic simulation of charm signals. See the text for more detail.

of minimum bias collisions corrected for pile-up, and to multiply by the minimum bias cross section $\left(\sigma_{\mathrm{MB}}\right)$. This cross section is derived from the $\sigma_{\mathrm{V} 0 \mathrm{AND}}$ cross section measured with the Van Der Meer scan method [18], V0AND corresponding to coincidence signals in the two VZERO detectors. The $\sigma_{\mathrm{V} 0 \mathrm{AND}} / \sigma_{\mathrm{MB}}$ ratio is the percentage of minimum bias events with the L0 trigger input fired and with V0AND condition. Its value is 0.87 and remains stable within $1 \%$ over the analyzed data taking period. This leads to $\sigma_{\mathrm{MB}}=71.4 \pm 0.7$ (stat. $) \pm 7.1$ (syst.) $\mathrm{mb}^{1}$.

\subsection{Experimental results}

The production differential cross section of muons from charm and beauty decay as a function of $p_{\mathrm{t}}$ in the acceptance of the muon spectrometer, in $p$ - $p$ collisions at $\sqrt{s}=7 \mathrm{TeV}$ is depicted in Fig. 6 (upper panel). The statistics corresponds to an integrated luminosity of $3.49 \mathrm{nb}^{-1}$. Statistical uncertainties are negligible (smaller than the symbols) and systematic errors (boxes) vary from $30 \%$ (low $p_{\mathrm{t}}$ ) to $20 \%$ (high $p_{\mathrm{t}}$ ). The systematic uncertainty on $\sigma_{\mathrm{MB}}$ (about $10 \%$, see sub-section 3.4 ) is not displayed. The various sources of systematic uncertainties that we have considered are shown, as a function of $p_{\mathrm{t}}$, in Fig. 6 (lower panel). The systematic uncertainty includes:

- alignment: $2 \% \times p_{\mathrm{t}}$ (blue);

- background subtraction: from $20 \%$ to $7 \%$ as $p_{\mathrm{t}}$ increases from $2 \mathrm{GeV} / \mathrm{c}$ to $6.5 \mathrm{GeV} / \mathrm{c}$ (red);

- detector response: $5 \%$ (green);

\footnotetext{
${ }^{1}$ Note that $\sigma_{\mathrm{V} 0 \mathrm{AND}}$ has been recently updated and the final value of $\sigma_{\mathrm{MB}}$ is $62.3 \pm 0.4($ stat. $) \pm 4.3($ syst. $)$ mb.
} 
- Monte-Carlo description: 20\% (magenta).

Finally, the resulting systematic uncertainty (yellow band) is the quadratic sum of the various individual contributions.

It is worth mentioning that the $p_{\mathrm{t}}$ reach (Fig. 6) is restricted to $6.5 \mathrm{GeV} / \mathrm{c}$ due to the limited statistics in the realistic simulations needed for acceptance and efficiency correction. The data are compared to Fixed Order Next-to-Leading Log predictions [19] (yellow band, upper panel of Fig. 6). An overall good agreement between data and model predictions is evidenced within errors.
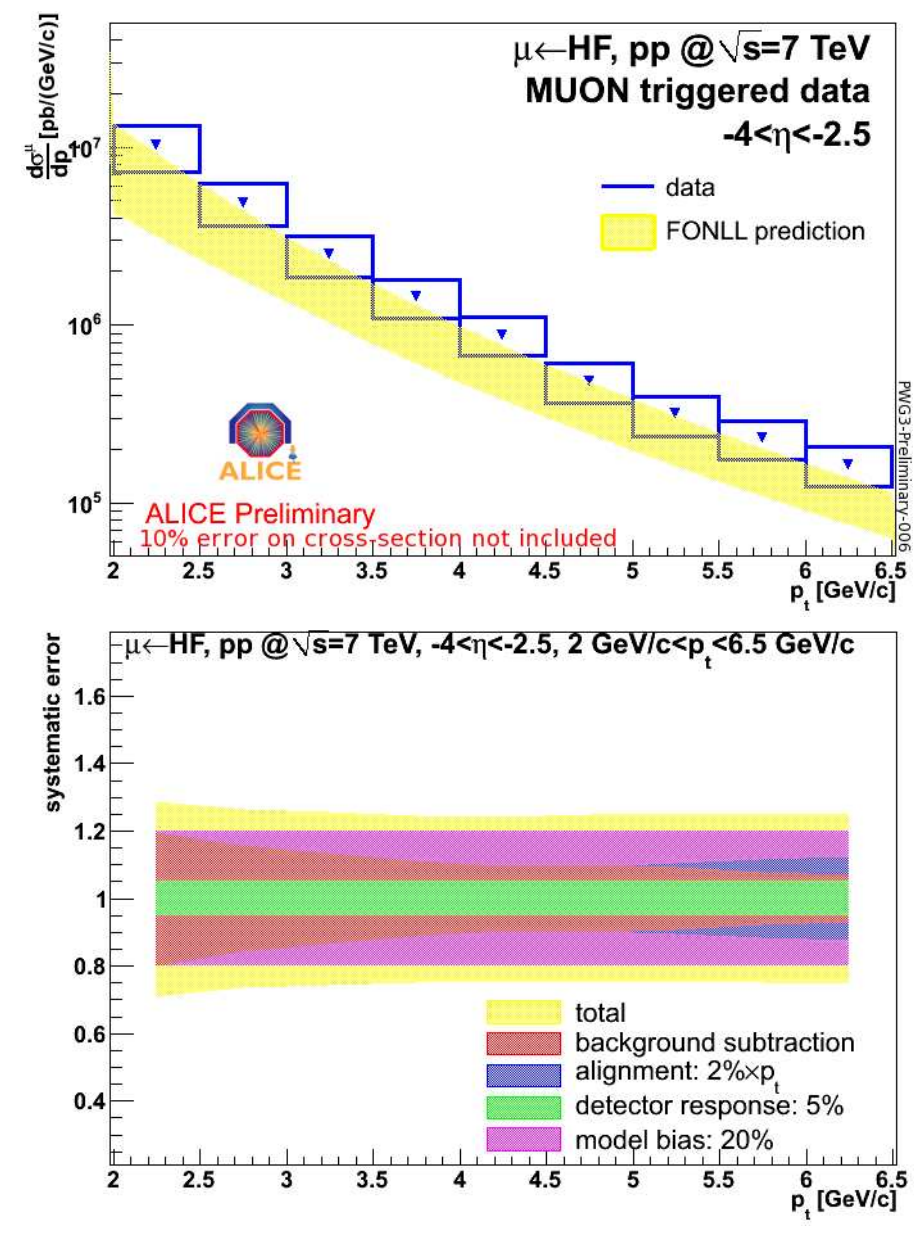

Figure 6: Upper panel: Inclusive production differential cross section of muons from heavy flavour decay in $p-p$ collisions at $\sqrt{s}=7 \mathrm{TeV}$ (symbols). The results have been obtained in the forward rapidity region $(-4<\eta<-2.5)$; the data are compared to FONLL predictions. Lower panel: Systematic uncertainties as a function of $p_{\mathrm{t}}$. See the text for more detail.

The analysis of the whole statistics collected in 2010 will allow to extend the $p_{\mathrm{t}}$ range to about $20 \mathrm{GeV} / \mathrm{c}$ and should allow to separate charm and beauty components by means of a combined fit. Going further, it should be possible to convert the charm and beauty production differential cross sections at muon level to the corresponding hadron production differential cross section. This is described in the next section. 


\section{Performance of the ALICE muon spectrometer for the measurement of the $B$-hadron and $D$-hadron production cross sections: simulation results}
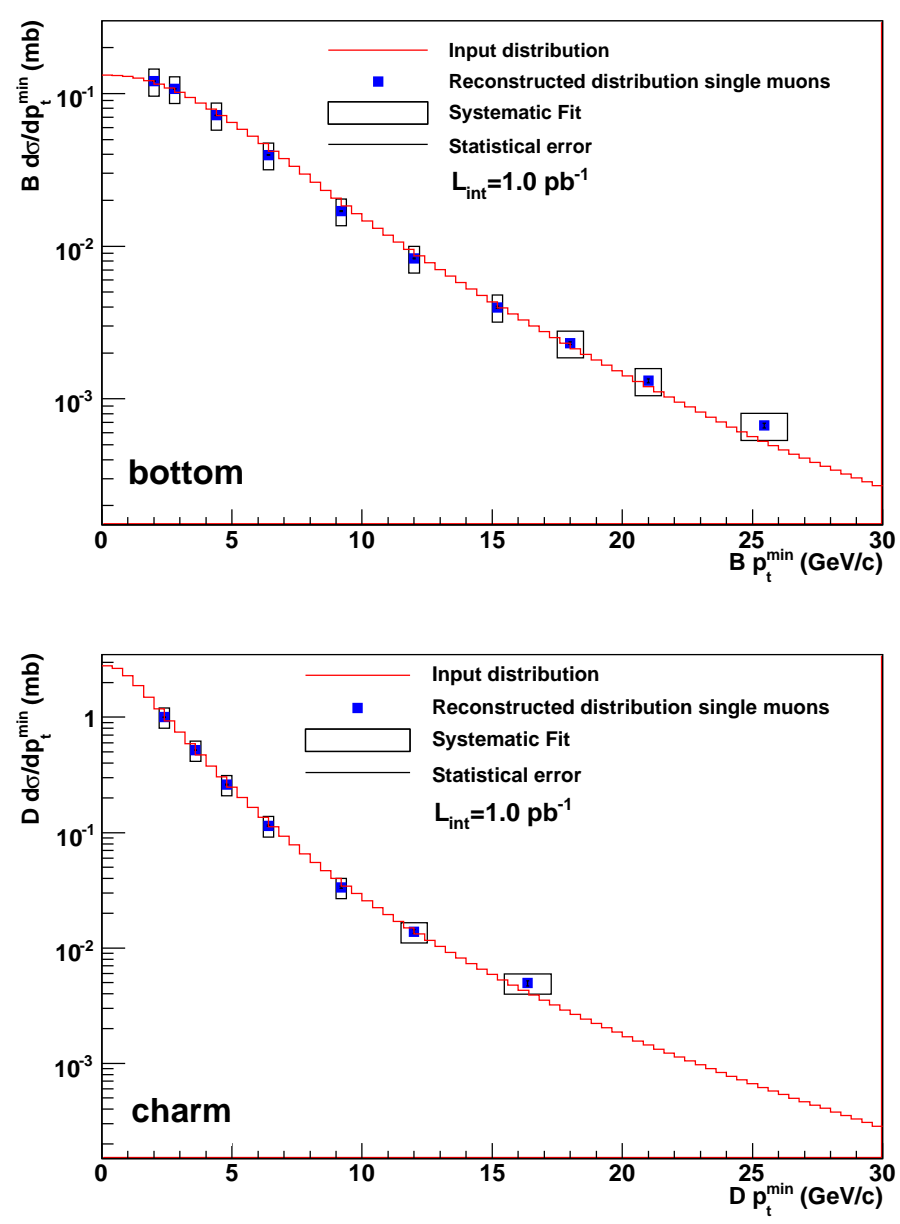

Figure 7: $B$-hadron (upper panel) and $D$-hadron (lower panel) inclusive production differential cross sections in $p$ - $p$ collisions at $\sqrt{s}=14 \mathrm{TeV}$ for an integrated luminosity of $1.0 \mathrm{pb}^{-1}$. The results are shown in the forward region $(-4.0<\eta<-2.5)$. See the text for more details.

The performance of the ALICE muon spectrometer for the measurement, via single muons, of the $B(D)$-hadron inclusive production differential cross section has been evaluated in $p$ - $p$ collisions at $\sqrt{s}=14 \mathrm{TeV}[20,21]$ by means of realistic simulations. The principle is first to estimate the muon yield from heavy flavour decay from the total transverse momentum $\left(p_{\mathrm{t}}\right)$ distribution as discussed in section 3 . Then, beauty and charm muon components are unraveled via a combined fit which includes predicted shapes of the different components ${ }^{2}$. This done according to: $(T-B) \times\left(f_{c}^{\mu}+\right.$ $\left.R \times f_{b}^{\mu}\right) . f_{c}^{\mu}$ and $f_{b}^{\mu}$ are the normalized shape functions for charm and beauty, respectively. $T$ is the total number of muons after background subtraction, $B$ is the beauty amplitude and $R$ is the ratio of beauty over charm amplitude. Therefore, one is left with two free parameters $R$ and $B$. Finally, the

\footnotetext{
${ }^{2}$ Note that charm and beauty components have never been disentangled with this method in the past.
} 
$B$-hadron and $D$-hadron production cross sections are determined after corrections for efficiency, luminosity, branching ratios and decay kinematics by using the method initially developed by the UA1 collaboration [22]. Figure 7 shows the reconstructed $B$-hadron (upper panel) and $D$-hadron (lower panel) cross sections as a function of $p_{\mathrm{t}}^{\mathrm{min}}$. The statistics corresponds to an integrated luminosity of $1.0 \mathrm{pb}^{-1}\left(\mathrm{t}=10^{6} \mathrm{~s},\left\langle L>=10^{30} \mathrm{~cm}^{-2} \mathrm{~s}^{-1}\right)\right.$. The measurement of the $B$-hadron $(D$ hadron) cross section can be performed over a large $p_{\mathrm{t}}$ range going from about $2 \mathrm{GeV} / \mathrm{c}$ to $25 \mathrm{GeV} / \mathrm{c}$ $(16 \mathrm{GeV} / \mathrm{c})$ with statistical errors lower than $10 \%$ at high $p_{\mathrm{t}}$. The expected systematic uncertainties, mainly due to the fit assumptions, are about $20 \%$. A similar analysis has been successfully carried out in the dimuon channel [21] and a nice agreement between the different channels has been evidenced.

\section{Conclusion and outlook}

We have presented the first measurement of the inclusive $p_{\mathrm{t}}$-differential production cross section of muons from heavy flavour decay in $p$ - $p$ collisions at $\sqrt{s}=7 \mathrm{TeV}$, in $2<p_{\mathrm{t}}<6.5 \mathrm{GeV} / \mathrm{c}$, using the excellent capabilities of the ALICE muon spectrometer. Despite the present large uncertainties, the agreement between data and FONLL pQCD calculations is rather good. We are presently working at having a better control on the different sources of systematics in order to reduce the corresponding uncertainties. These results will provide a very important baseline for the analysis and understanding of $\mathrm{Pb}-\mathrm{Pb}$ data. The analysis of the whole statistics recorded in 2010 is ongoing. This will allow to extend the upper limit of the $p_{\mathrm{t}}$ range to about $20 \mathrm{GeV} / \mathrm{c}$ and to perform $p_{\mathrm{t}}$-differential measurements in several $\eta$ bins. We will further separate the charm and beauty components and measure the production cross sections at the hadron level.

The LHC delivered its first $\mathrm{Pb}-\mathrm{Pb}$ beams at a center of mass energy per nucleon pair $\sqrt{s_{N N}}$ $=2.76 \mathrm{TeV}$ in November 2010. This will open a new era for studying the properties of strongly interacting matter in extreme conditions of temperature and density. The full analysis of heavy flavour production in the single muon channel is in progress. The measurement should allow the study of the central to peripheral ratio $\left(R_{\mathrm{CP}}\right)$ and nuclear modification factor $\left(R_{\mathrm{AA}}\right)$.

\section{References}

[1] F. Carminati et al., ALICE Collaboration, J. Phys. G: Nucl. Part. Phys 30 (2004) 1517.

[2] M.L. Mangano, P. Nason, G. Ridolfi, Nucl. Phys. B 373 (1992) 295.

[3] A. Dainese, ALICE Collaboration, Eur. Phys. J. C49 (2007) 135.

[4] Z. Conesa del Valle et al., Phys. Lett. B 663 (2008) 202.

[5] B. Alessandro et al., ALICE Collaboration, J. Phys. G: Nucl. Part. Phys 32 (2006) 1295.

[6] K. Aamodt et al., ALICE Collaboration, Eur. Phys. J. C 65 (2010) 111.

[7] K. Aamodt et al., ALICE Collaboration, Eur. Phys. J. C 68 (2010) 89.

[8] K. Aamodt et al., ALICE Collaboration, Eur. Phys. J. C 68 (2010) 345.

[9] K. Aamodt et al., ALICE Collaboration, Phys. Rev. Lett. 105 (2010) 072002.

[10] K. Aamodt et al., ALICE Collaboration, Phys. Rev. D 82 (2010) 052001. 
[11] K. Aamodt et al., ALICE Collaboration, Phys. Lett. B 693 (2010) 53.

[12] K. Aamodt et al., ALICE Collaboration, Phys. Rev. Lett. 105 (2010) 252301

[13] K. Aamodt et al., ALICE Collaboration, Phys. Rev. Lett. 105 (2010) 252302.

[14] K. Aamodt et al., JINST 3 (2008) S08002.

[15] V. Blobel and C. Kleinwort, arXiv:hep-ex/0208022.

[16] P. Z. Skands, Phys. Rev. D 82 (2010) 074018.

[17] A. Moraes, ATLAS Note ATL-COM-PHYS-2009-119 (2009).

[18] S. Van der Meer, ISR-PO/68-31, KEK68-64.

[19] M. Cacciari, S. Frixione and P. Nason, JHEP 0103 (2001) 006, [arXiv:hep-ph/0102134].

[20] L. Manceau et al., Eur. Phys. J. C 62 (2009) 9.

[21] X. Zhang et al., Chinese Phys. C 34 (09) (2010) 1538.

[22] C. Albajar et al., Phys. Lett. B 213 (1988) 405, Phys. Lett. B 256 (1991) 121. 